\title{
L'eau naturelle superficielle courante
}

\author{
Flowing natural surface water
}

\author{
PAR M. CHARTIER, \\ professeun chaRgÉ de REGHERGHES, DOCTEUR EN GÉogRaPHE
}

\begin{abstract}
Pendant des siècles, l'eau naturelle superficielle courante a été mobilisée comme facteur des productions agricoles et comme source d'énergie; de nos jours, sous la pression des besoins nouveaux et de techniques nouvelles, cette eau est promne au rang de matière première.

En dépit de son apparente simplicité de composition, l'eau naturelle n'est pas pure : c'est une substance complexe dont les caractères, non immuables, dépendent des données géologiques, lithologiques et climatiques. Sa connaissance ne peut être acquise qu'après de multiples mesures et analyses. L'auteur expose brièvement ce qu'est cette ean naturelle et comment elle se présente.
\end{abstract}

Le géographe potamologue que je suis considère comme un fait d'importance majeure que le Comité Technique de la S.H.F. consacre l'une des séances de cette session plénière aux problèmes de la qualité des eaux. Certes, il n'en est point surpris; il sait que les ingénieurs utilisateurs se préoccupent du problème de l'eau au triple point de vue de la quantité, de la qualité et du rejet et que ce problème accroît d'une manière sensible les dépenses, donc le prix de revient. Il n'ignore pas que, présentement, l'implantation d'une usine nouvelle dépend autant de Ja proximité des sources de matières premières ou de production, d'une bonne desserte par les moyens de transports appropriés, d'une réserve suffisante de main-d'œuvre qualifiée on non, que de la disponibilité en quantité et en qualité de l'eau nécessaire aux besoins de l'industrie et des personnes. Un seul exemple nous permettra d'illustrer cette nécessité d'avoir à son service un volume considérable d'eau : c'est l'équipement hydraulique des usines Simca à Poissy; cet équipement comprend un château d'eau de $75 \mathrm{~m}$ de haut avec un réservoir de $1500 \mathrm{~m}^{3}$ et une station de pompage dans la Seine avec une installation de traitement capable de débiter $2500 \mathrm{~m}^{3}$

\begin{abstract}
Man has been using natural surface water on the land as a sonrce of power for centuries, and as a result of the new requirenents and technical developments of this modern age it has now become an important raw material. Natural water is by no means pure, despite its apparently simple make-llp. On the contrary, it is a complex substance, with variable properties depending on geological and lilhological conditions and climate; the identification of its properties involves much analysis and very comprehensive measurements. The anthor briefly describes nafural water and discusses the form in which it occurs.
\end{abstract}

à l'heure, c'est-à-dire de satisfaire la consommation domestique d'une ville de 250000 habitants.

En un temps "où l'on utilise de plus en plus les eaux courantes pour des fins variées » [1], l'intérêt scientifique de l'hydrologie fluviale ćclate aux yeux en fonction de l'ampleur de sa contribution aux activités humaines.

\section{I. - DE LA MOBILISATION DE L'EAU NATURELLE A SA PROMOTION AU RANG DE MATIÈRE PREMIERE}

Durant les siècles de ce qu'on a appelé "l'ère agricole et éleveuse »[2], les villes, souventefois, et les fabriques, en majeure parlie, étaient localisées le long des rivières pérennes dans lesquelles elles se procuraient l'eat pour l'alimentation, l'eau pour la navigation, l'eau pour l'énergie, l'eau pour les usages industriels, l'eau pour l'évacuation des déchets. A dire vrai, après l'invention des machines de fabrication que mouvaient des chutes d'eau et avant l'invention des 
machines à vapeur, l'eau était mobilisée, en premier lieu, par l'agriculture (puisque l'eau est un des facteurs essentiels des productions agricoles, végétales et animales), pour l'arrosage des champs, des vergers et des prés, notamment dans les régions de climat sec; en dehors de l'activité agricole, elle l'était comme source naturelle d'énergie par les fabriques et les manufactures, première forme de concentration en matériel, en ouvriers et en capitaux, aussi bien que par les industries domestiques, forme la plus répandue alors d'une activité artisanale semi-paysanne, semi-industrielle. C'est d'ailleurs l'insuffisance des cours d'eau pour actionner les roues à aubes et les difficultés techniques et financières poux construire des réservoirs qui stimulèrent l'imagination el l'ingéniosité des gens de métier et furent à l'origine de l'invention de la machine à vapeur. Mais, avec la révolution technique, l'utilisation de l'eau par l'industrie d'abord, par l'agriculture moderne actuellement, augmenta considérablement et continue d'augmenter, de telle sorte qu'en maintes régions, les demandes croissantes menacent de devenir supérieures aux ressources : en Graude-Bretagne, les demandes en eau par l'industrie auront doublé entre 1938 et 1968; en Allemagne Fédérale, l'expansion industrielle de la Ruhr sera limitée par le manque d'eau; aux Etats-Unis d'Amérique du Nord, le niveau des eaux souterraines dans lesquelles puisent les gros centres industriels s'est abaissé de près de $40 \mathrm{~m}$. En France, chacun connaît des exemples. Le problème est devenu universel.

Ainsi, ce corps naturel commun, dont la présence est si familière à l'homme, mais dont le rôle est si fondamental que la symbolique des Anciens en fit un des quatre éléments, a pu être considéré pendant longlemps, sous nos climats au moins, comme une chose sans valeur, d'apparence inépuisable, qu'on pouvait gaspiller. Mais l'essor de notre civilisation, que certains penseurs français qualifient de «mécanique » ou de «technicienne», a assuré la promotion de l'eau au rang de matière première, c'est-à-dire d'un «produit brut nécessaire pour l'élaboration, par le travail industriel, d'objets fabriqués » [3] ; promotion qui résulte de la pression des besoins nouveaux et de la mise an point de techniques nouvelles. De cette économie industrielle procède le développement des agglomérations urbaines et, par suite, du rôle de l'eau comme facteur d'hygiène publique [4]. Dès lors, déjà pénétré de l'idée que, en biologie, rien ne peut remplacer l'eau, de surcroit convaineu de la valorisation qu'a prise, dans la civilisation moderne, ce liquide originellement bon marché, le lecteur averti de la revue d'Allemagne Fédérale Das Parlament, comprend que l'eau puisse être appelée « das weisse Gold», l'or blanc.

Faut-il croire que nous découvrons le pro- blème de l'eau? Si, quantitativement, les ressources disponibles en eau utilisable commencent, en fonction de l'activité économique et de l'évolution sociale, à révéler parfois une situation critique qui inquiète les responsables de la cité, il semble que l'attention se soit attachée très tôt à la qualité de l'eau; ce respect qu'on avait pour elle se traduisait par le culte dont elle était l'objet dans le polythéisme antique ou par l'intérêt que, déjà, l'Administration lui portait : la croyance à la purification morale par l'eau impliquait la pureté de l'élément liquide naturel; en Gaule, les druides veillaient à ce que l'eau des sources ne fût point souillée; à Rome, sous la République, des fonctionnaires, les curatores alvei Tiberis, étaient chargés de surveiller le cours du Tibre; la législation édictée qui assurait la propreté des fontaines prévoyait des peines sévères contre qui polluait leurs eaux: à Pergame, en cas d'infraction, l'homme libre pouvait voir confisquer ses biens et l'esclave pouvait se voir infliger une peine corporelle de deux fois cent coups de bâton à dix jours d'intervalle... Chez nous, l'aspect qualitatif n'était pas méconnu dans les années passées; cette eau naturelle brute, pompée dans la rivière et présumée propre, ne laissait pas d'avoir des inconvénients, par exemple, dans les circuits de réfrigération, par les dépôts, l'entartrage ou la corrosion. Mais, cette eau de rivière que l'on feignait de croire propre devient, par le fait de l'activité humaine, de plus en plus médiocre : le riverain, le pêcheur, l'utilisateur le constatent; le spécialiste se plaint de ce que son traitement pose des problèmes délicats à résoudre et impose des dépenses d'épuration ou d'adoucissement très lourdes. La détérioration de ses qualités naturelles ne saurait s'accuser sans nuire gravement à la population, à l'agriculture et à l'industrie.

\section{II. - CE QU'EST L'EAU NATURELLE SUPERFICIELLE COURANTE}

Il me semble opportun de nous demander ce qu'est cette eau naturelle de rivière avant sa pollution par des déversements urbains et industriels et par des produits utilisés en agriculture.

L'eau qui nous intéresse est une eau fluviale, une eau courante continentale de surface. Contrairenent à la tradition, je me refuse à la nommer « eau douce», même par opposition à « eau salée », car j'estime que dire «eau douce», c'est prendre une option chimique : une eau est chimiquement «douce» lorsqu'elle a en solution une très faible teneur d'ions $\mathrm{Ca}^{++}$et $\mathrm{Mg}^{++}$, tandis qu'une eau est chimiquement « dure »lorsqu'elle est riche en ions $\mathrm{Ca}++$ et $\mathrm{Mg}^{+}+$au point 
de coaguler le savon et d'incruster à froid ou à chaud les récipients qui la renferment. Précisons au passage que, à un degré moindre, les ions $\mathrm{Fe}^{++}$et $\mathrm{Al}+++$ peuvent produire les mêmes effets de dureté [5] [6]. J'emploie donc les expressions d' « eau fluviale naturelle» ou d' " eau naturelle superficielle courante».

Cette eau provient d'une eau souterraine qui apparaît à la surface en un point nommé généralement, mais maintes fois improprement, «source». Elle n'est pas pure; de même que n'est pas pure l'eau météorique sans laquelle ne seraient présentes ni l'eau superficielle ni l'eau souterraine : l'eau météorique, comme l'eau naturelle la plus limpide, d'une source ou d'une rivière, renferme, à l'état dissous, des sels minéraux, divers gaz, même des matières organiques; elles peuvent aussi entrainer ou transporter par suspension des éléments solides minéraux, des matières organiques (débris microscopiques et submicroscopiques d'origine animale et végétale) et des matières organisées (micro-organismes vivants).

A son origine, l'eau de pluie présente une pureté voisine de celle de l'eau bidistillée. Toutefois, des mesures et calculs très sensibles d'échantillons ont révélé, dans les nuages, une concentration de l'ion $\mathrm{Na}^{\dagger}$ et de l'ion $\mathrm{Cl}$ - de l'ordre de $5.10^{-6} \mathrm{mg} / 1$, soit $5 / 1000 \mathrm{~g} / \mathrm{m}^{3}$ [7]. Près des côtes, les ions $\mathrm{Na}+$ et $\mathrm{Cl}$ - prédominent; à l'intérieur du continent, leur teneur peut changer avec les régions et les saisons. Les analyses actuelles, très fines, montrent la présence, dans l'eau météorique recueillie par un pluviomètre spécialement conçu et aménagé, de divers ions en provenance de l'Océan, du sol ou de la pollution de l'atmosphère; cette eau météorique a procédé au lessivage des couches de l'atmosphère contenant des aérosols en suspension : ainsi, sur le plateau de Beauce, à Magny-les-Hameaux (Seine-et-Oise) [8], nous avons remarqué une teneur plus grande que la normale en ions $\mathrm{K}+$ après épandage d'engrais potassiques et en ions $\mathrm{Ca}++$ après une période sèche. Mais, si elles sont sujettes à des écarts considérables, ces teneurs demeurent généralement faibles, généralement aussi plus fortes au début d'une averse et susceptibles d'être influencées (au cours d'une même averse) par la direction et la vitesse du vent: le 24 mai 1960 , lors de la pluie du matin, la teneur en ions $\mathrm{Ca}^{+}+$a varié de $0,030 \mathrm{mg} / \mathrm{l}$ à $8,40 \mathrm{mg} / 1$. Bien que son influence soit négligeable le plus souvent, infime même parfois, la composition de l'eau de pluie méritait quelque attention.

Au contact du sol, puis au cours du ruissellement superficiel ou du parcours souterrain qu'elle accomplit entre le point d'infiltration et l'exutoire naturel à la surface, cette eau acquiert des propriétés nouvelles et se charge d'éléments nouveaux qu'elle emprunte aux roches sur lesquelles ou dans lesquelles elle séjourne et chemine. En effet, entraince par gravité vers le bas, l'eau superficielle intervient à la fois dans les échanges d'énergie avec le sol, car elle transporte des calories, et dans de nombreuses réactions chimiques avec les substances du sol (dissolutions et précipitations), car elle lransporte du gaz carbonique en quantité variable. Cette eau traverse des couches qui la débarrassent des matériaux très fins pris à la surface, mais qui la chargent de sels solubles. Par suite, l'eau qui sourd d'une roche-magasin présente des caractéristiques physiques et chimiques qui modifient à leur tour, au cours du mélange, la composition de l'eau de surface. Ces caractéristiques difrèrent d'une émergence à l'autre et, pour une même émergence, peuvent différer d'un jour à l'autre. Citons quelques résultats de l'analyse de deux prélèvements (fig. 1) effectués le même jour, à trente minutes d'intervalle, en deux émergences des sources de la Voulzie, près de Provins (Seineet-Marne).

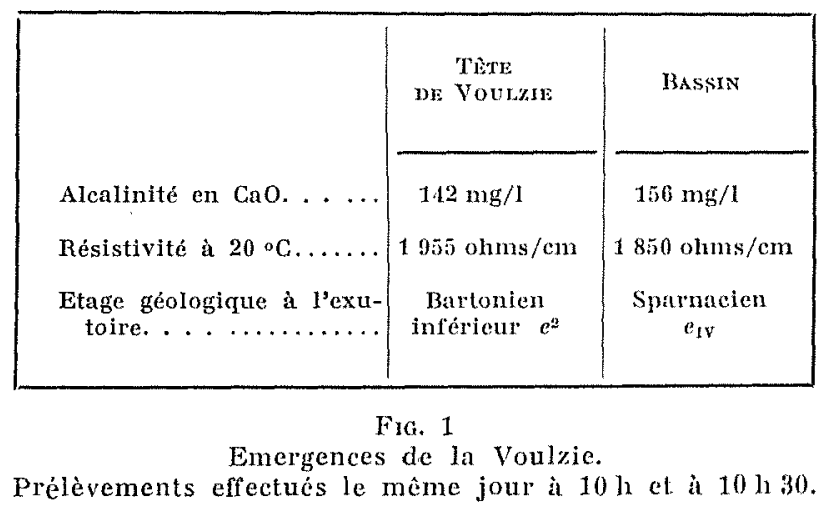

Par le seul fait de son ćcoulement en contact avec l'atmosphère et avec le sol, l'cau souterraine se trouve dans de nouvelles condilions ambiantes (température, pression, grandeur des surfaces de contact...) et hydrauliques (vilesse, agitation...) qui perturbent les équilibres et peuvent provoquer, par exemple, la précipilation du carbonate de calcium : c'est ainsi que les eaux de la source de la Dhuys à Pargny (Marne), dont l'alcalinité correspond à 250-260 $\mathrm{mg} / \mathrm{l}$ de $\mathrm{CaCO}_{3}$, abandonnent, après leur sorlic de terre, une partie de l'anhydride carbonique qu'elles renferment et commencent à déposer du $\mathrm{CaCO}_{3}$ au bout de 24 heures. Tout parcours de colle eau dans le lit d'un ruisseau ou d'une riviere ama pour conséquence d'altérer encore, a maintes reprises, ses propriétés initiales; le long du cours d'eau, les venues aflluentes superficielles ou souterraines, d'importance variable, visibles ou cachées, diversement chargées en sels dissous el en matières en suspension, interviennent à leur 
tour en fonction du volume de leur débit liquide. Les qualités physico-chimiques d'une eau naturelle superficielle courante, influencées par de nombreux facteurs naturels, relèvent des conditions locales et temporelles d'alimentation, des phénomènes qui concourent à l'écoulement, c'est-à-dire les données atmosphériques et cosmiques (précipitations liquides ou solides, température, ensoleillement...), les données géologiques et lithologiques, les données géomorphologiques et géobotaniques du bassin versant en amont $\mathrm{du}$ point de prélèvement avec leurs conséquences (érosion, dissolution, transport). Elles relèvent aussi de conditions hydro-biologiques : un ensemble d'êtres microscopiques végétaux et animaux flottent au sein de l'eau, passivement ou non, et forment le potamoplancton qui se divise en phytoplancton (composé de végétaux chlorophylliens aquatiques) et en zooplancton (dont les êtres se nourrissent de phytoplancton). Le phytoplancton seul nous intéresse ici, puisque lui seul modifie les qualités de l'eau dans laquelle il vit; les algues qu'il comprend synthétisent, à l'aide de l'énergie de la lumière, les matières organiques nécessaires à leur vie; elles sont autotrophes, c'est-à-dire qu'elles sont \& capables de trouver dans des composés minéraux et notamment dans $\mathrm{CO}_{2}$ le carbone dont elles ont besoin pour leurs synthèses » [9]. D'autres éléments, qu'elles puisent dans l'eau et les sels dissous, s'ajoutent au carbone: hydrogène, oxygène, azote, soufre. Elles prennent le carbone au $\mathrm{CO}_{2}$ libre dans l'eau et, pour ne pas que soit rompu l'équilibre; chimique, une partie du $\mathrm{CO}_{2}$ semi-combiné des bicarbonates est libéré naturellement, d'où précipitation des carbonates insolubles correspondants et diminution de la teneur en carbonates et bicarbonates alcalino-terreux. Il en résulte une modification des propriétés chimiques de l'eau, modification due aux algues planctoniques, dont il est avéré que l'importance en hydrochimie est appréciable, mais encore insuffisamment connue.

En dépit de son apparente simplicité d'e composition, l'eau naturelle est donc une substance très complexe; c'est un mélange naturel, homogène ou hétégorène selon que cette eau possède ou ne possède pas les mêmes propriétés en tous ses points.

\section{III. - COMMENT SE PRESENTE CETTE EAU NATURELLE SUPERFICIELLE COURANTE}

Lorsqu'elle est vue sous une faible épaisseur, en été ou en période sèche, sur un fond rocheux ou caillouteux, surtout si elle est issue d'un terrain cristallin ou du réseau aquifère qui existe dans le calcaire à entroques, par exemple, l'eau naturelle paraît limpide, incolore ou du moins présente la couleur du fond du lit. En réalité, ne nous y trompons pas : une eau peut être limpide à l'œil nu sans être parfaitement transparente; un faisceau lumineux qui la traverse révèle qu'elle n'est pas optiquement vide. Le trouble mis en évidence est dû̀ à la présence de corpuscules très fins dispersés dans le liquide : ce sont des matières colloïdales en suspension, d'origine minérale ou organique; la plupart du temps, il s'agit de particules d'argile. Après un certain parcours, ces eaux finissent par perdre leur limpidité et ce parcours sera très bref s'il survient une pluie violente ou prolongée. Au reste, Belgrand a distingué trois états de limpidité pour l'eau de rivière: l'eau peut être clcitre, louche ou trouble selon qu'un disque blanc d'un $\mathrm{dm}^{2}$, plongé sous une épaisseur de $50 \mathrm{~cm}$, se voit nettement ou confusément ou cesse d'ètre visible.

Rivières et torrents offrent des teintes extrêmement variées. Leur couleur est un caractère organoleptique qui, depuis longtemps, a été remarqué par les hommes : sur tous les continents, des cours d'eau portent des noms inspirés par leur teinte dominante. L'origine de la coloration de l'eau n'est pas simple; nous pourrons nous attacher à ce problème de la couleur lors d'une autre session, si vous le désirez; la couleux réelle est communiquée à l'eau par les substances en solution et la couleur apparente, par les matières solides en suspension. Outre des phénomènes physiques et le débit solide, intèrviennent fréquemment dans la coloration de l'eau des substances dissoutes et des substances colloïdales selon des processus chimiques complexes et parfois mal connus. Une eau ferrugineuse, contenant de l'oxyde ferreux qui, à l'air, donne de l'oxyde ferrique, prend une coloration due à un trouble rougeâtre; les eaux granitiques du Morvan sont souvent brunes, d'une couleur allant $d u$ jaune ambre au brun jaunâtre; ces dernières eaux ont une réaction acide marquée, un $\mathrm{pH}$ inférieur à 6 , une teneur en sels de $\mathrm{Ca}++$ et de $\mathrm{Mg}^{++}$très faible, une oxydabilité d'autant plus élevée que le $\mathrm{pH}$ est bas, tous ces caractères étant liés à la présence d'acides humiques qui les colorent.

Des éléments biologiques sont susceptibles d'ajouter encore à la complexité des facteurs capables de déterminer la couleur d'une eau naturelle. Citons quelques exemples entre mille peut-ĉtre: les algues vertes, telle Pediastrum Clathratum, ou les algues de teinte brune, telle une Diatomée: Melosira dans la Seine ou la Marne [10], ou encore des ferro-bactéries, plus localement.

Il est aisé de déterminer directement la limite de transparence d'une eau naturelle : la technique la plus simple consiste à descendre un disque blanc dans le lit du cours d'eau et à mesurer la profondeur à laquelle ce disque, appelé dis- 
que de Secchi, disparait. Dans l'Yonne, cette profondeur a varié, en 1962, entre 1,60 $\mathrm{m}$ et $0,03 \mathrm{~m}$; dans la Marne, à Joinville-Saint-Maur, entre $1,50 \mathrm{~m}$ et $0,10 \mathrm{~m}$; j'ai noté, dans un rapport de 1928, que, en période d'étiage, la limite de transparence à Joinville était à $2,50 \mathrm{~m}$; de nos jours, elle n'est jamais à une profondeur supérieure à $1,50-1,60 \mathrm{~m}$. Cette appréciation de la limite de transparence exprime une évaluation grossière du trouble de l'eau examinée; c'est un élément de la description de la rivière en un point donné, avec la couleur, l'état hydrologique, la nature du lit et des berges... Ce trouble naturel ou débit solide est, pour une part appréciable, formé de matériaux fins ou très fins qui sont transportés en suspension dans l'eau tant que la vitesse est suffisante pour provoquer les phénomènes de turbulence; les particules qui composent ces matériaux vont $d u$ sable aux argiles, c'est-à-dire, d'après l'échelle granulométrique de l'«American Geographical Union», de $2000 \mu$ à $0,24 \mu$.

La charge minérale solide naturelle de la lame d'eat dans la section mouillée peut comprendre deux parties que nous dénommerons la charge endogène et la charge exogène. La charge endogène ou charge du lit provient des dépôts alluviaux existant préalablement dans le lit mineur ou lit apparent du cours d'eau, mais remis en suspension, ou bien de l'érosion des berges. La charge exogène, ou charge des versants, est constituée par les particules déchaussées et entrainées par les eaux de ruissellement et provenant de l'usure des versants, c'est-à-dire des surfaces hors du réseau. La mesure précise de cette charge solide minérale est toujours délicate et les données numériques valables sont rares.

Dans Je bassin de la Seine, cette charge totale n'atteint ou ne dépasse qu'exceptionnellement $100 \mathrm{mg} / \mathrm{l}$, c'est-à-dire $100 \mathrm{~g} / \mathrm{m}^{3}$. Tel flot de crue de l'Yonne en aval de l'Armancon (fig. 2) avait une charge solide totale, recueillie par filtration sur membrane cellulosique, de $93,4 \mathrm{mg} / \mathrm{l}$, se répartissant en $81,8 \mathrm{mg} / \mathrm{l}$ de matières minérales et $11,6 \mathrm{mg} / 1$ de matières organiques et organisées, Quinze jours plus tard, la crue étant terminée et la limite de visibilité étant passée de $0,30 \mathrm{~m}$ à $1,30 \mathrm{~m}$, la charge totale n'était plus que de $7,7 \mathrm{mg} / 1$ dont $3,8 \mathrm{mg} / 1$ de matières minérales et $3,9 \mathrm{mg} / 1$ de matières organiques et organisées. Dans le premier cas, la charge minérale représentait $77 \%$ de la charge totale et, dans le deuxième cas, un peu moins de $50 \%$. Il importe donc vraiment de tenir compte de cette charge organique et organisée naturelle dont la proportion peut être considérable, puisque l'eau est un milieu biologique et que, en plus des matières minérales et des débris organiques inertes, elle transporte des micro-organismes du potamoplancton.

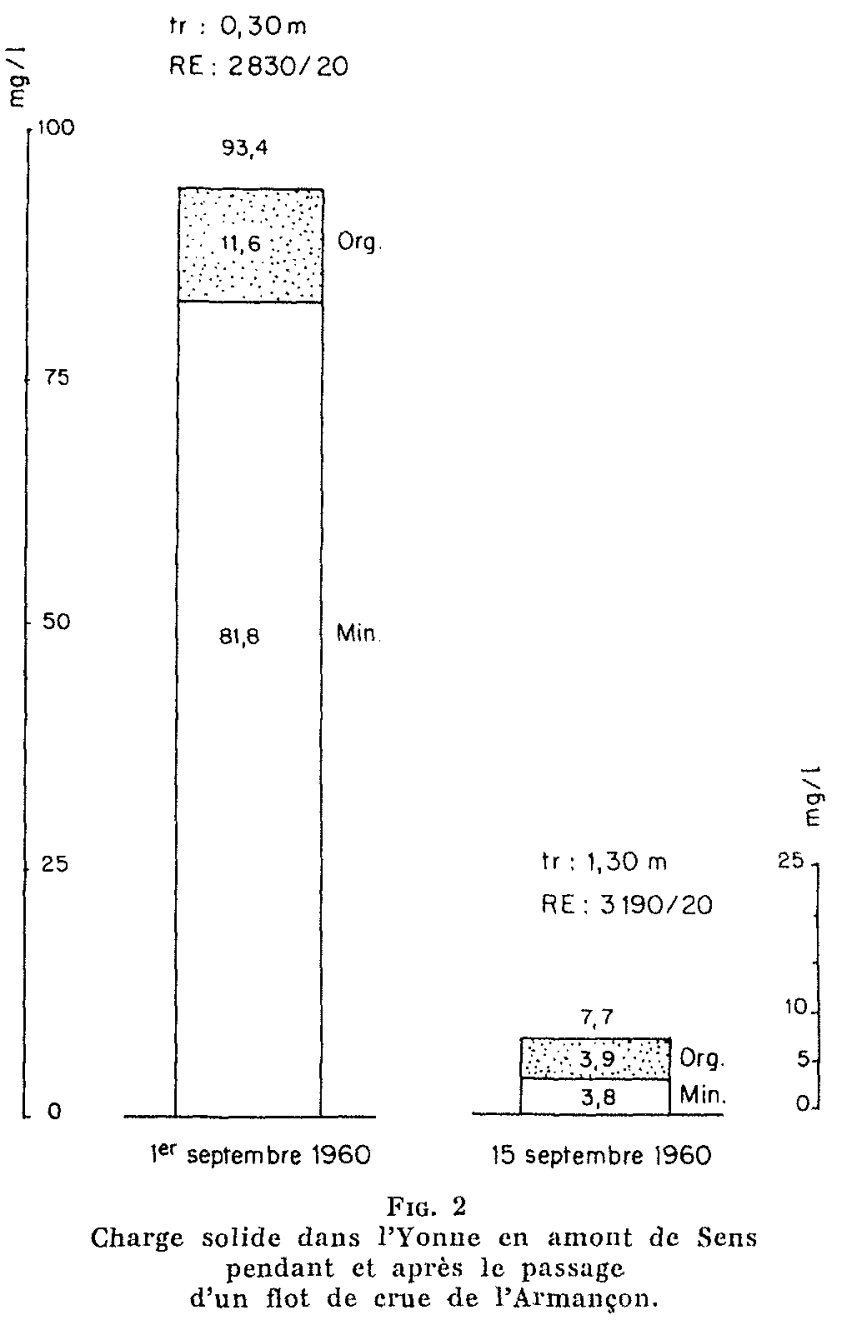

Pour intéressantes qu'elles soient, ces observations et mesures ne suffisent pas à l'hydrologue : il désire s'approcher de la meillcure connaissance d'une eau naturelle courante de surface qu'il se propose de caractériser en vue d'un classement dans une certaine catégorie, afin de prévoir ses «effets» et son utilisation ultérieure. Il cherche à déterminer ses qualités physico-chimiques, voire minéralogiques et biologiques, c'est-à-dire en quelque sorte à établir la fiche signalétique d'un flot dans telle riviere, tel jour, à telle heure, en tel endroit, dans telles conditions météoro-hydrologiques. Ces caractères ne sont pas, naturellement, immuables; mais les traits majeurs demeurent. Le potamologue agit, en somme, à l'image de l'anthropologue qui procède, à l'étude, dans leurs caractòres corporels, des groupes humains, c'est-à-dire des races et des lignées.

Plusieurs remarques s'imposent d'ailleurs: d'une part, dès l'abord, il peut apparaître qu'il devient particulièrement malaisé, dans nos régions, de trouver de l'eau de surface "vierge", de l'eau qui a totalement échappé aux transfor- 


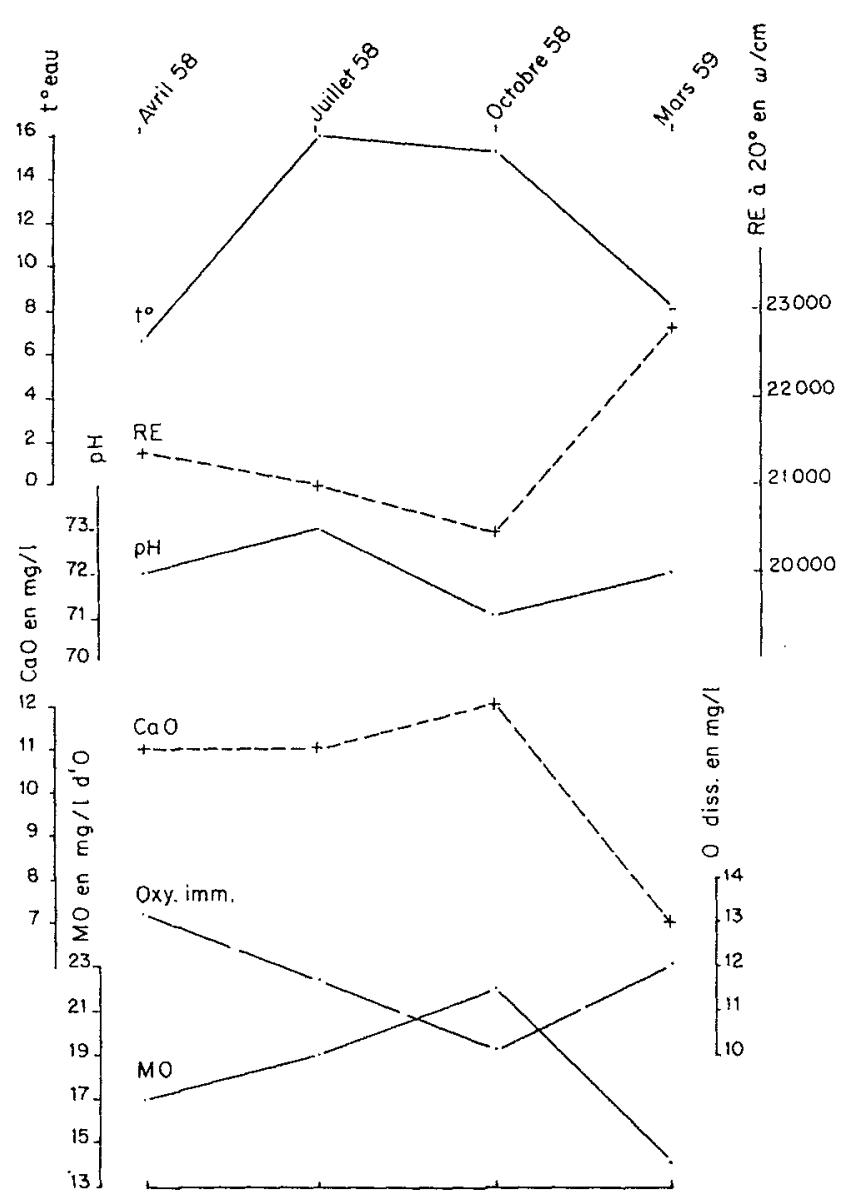

Fig. 3

Quelques mesures et dosages dans l'Yonne supérieure (une saison chaude et une saison froide).

mations que l'homme a apportées aux conditions initiales offertes par la nature; cependant, des facteurs constants subsistent (géologie, lithologie, morphologie, climat...) dont l'influence demenre prépondérante, parfois même encore absolue, dans la partie amont du bassin supérieur. C'est pourquoi la possibilité existe de' se livrex à des observations sérieuses, dans des conditions favorables, de l'eau issue d'un milieu géographique naturel qui est une combinaison d'éléments physiques et biologiques non altérés par I'homme; mais l'opérateur devra se livrer à une prospection attentive du bassin d'alimentation. D'autre part, les enquêtes par mesure et dosages ne seront pas appuyées sur une seule analyse ponctuelle et temporelle; J'analyse n'est qu'un moyen de contribuer à la connaissance de l'eau et ne peut être confondue avec elle : "analyser un ćchantillon d'eau, c'est fixer sur le papier l'expression fugitive d'un moment» (A. Guillerd). En un même point, mesures et dosages seront effectués à des intervalles réguliers, sur une longue période, dans les mêmes conditions, plus fréquemment au moment des extrêmes. Des précautions minutieuses seront prises, aussi bien pour le prélèvement que pour la conservation pendant le transport des échantillons; c'est fondamental, car, sans ces précautions, "l'hydrologie en bouteille est une duperie », dit avec vigueur un éminent spécialiste, M. Coutris. En outre, la connaissance de l'eau naturelle ne sera acquise qu'après la discussion de maints résultats d'analyses renouvelées et l'étude critique de la situation hydrologique lors de chaque prélèvement. L'hydrologue ne peut done s'approcher de la connaissance de l'eau naturelle qu'après avoir appliqué un programme d'étude à longue échéance. Par exemple, je puis vous dire que le titre hydrotimétrique de la Seine en amont de Paris est de $20^{\circ}$, que la teneur en sulfates (en $\mathrm{SO}_{3}$ ) est de $10 \mathrm{mg} / \mathrm{l}$, que la teneur en $\mathrm{SiO}_{2}$ est de $6 \mathrm{mg} / \mathrm{l}$, parce que ces valeurs expriment les moyennes de plusieurs milliers de résultats obtenus toujours dans les mêmes conditions.

Mais notre documentation sur la qualité des eaux naturelles est fort déficiente; vous le savez; d'autres vous le diront. La Commission d'Hydrologie Fluviale du Comité National de Géographie, laquelle est présidée par M. Maurice Pard'é et vice-présidée par MM. Marc Henry et CharlesP. Péguy, a formulé le vou que des études systématiques des caractères des eaux naturelles soient entreprises en France et que tous les renseignements soient centralisés : $M$. le Président jugera de l'opportunité d'associer le Comité Technique au voen des Géographes; chacun s'en réjouira s'il a la certitude que cette démarche n'est pas un coup d'épée... dans l'eau.

L'examen physico-chimique d'une eau naturelle peut requérix un grand nombre de recherches. Pour être bref, nous avons délibérément procédé à un choix qu'illustrera le tableau suivant (fig. 3). Il s'agit d'une eau naturelle, puisqu'elle est prélevée dans l'Yonne, au milieu du lit, à une quinzaine de kilomètres en aval du barrage de Pannesière-Chaumard. Ces valeurs ne sont pas des moyennes, hélas! Ce sont des résultats ponctuels de quatre prélèvements faits trimestriellement au cours de la période d'avril 1958 à mars 1959 , donc à raison de deux prélèvements pour chacune des saisons, chaude et froide.

La première des données est la valeur de la température de l'eau dans la rivière. On sait quelle est l'importance de la connaissance de celte grandeur physique à laquelle on ne porte généralement pas une attention suffisante. Consćquence directe et immédiate du climat, clle reflète la température locale et suit les variatious saisonnières. La température de l'eau naturelle intlue sur le pH, sur l'ionisation, sur la solubilité des gaz, sur la viscosité, sur la densité de microflore et microfaune dans la rivière.

$L e p H$ de l'eau exprime l'acidité ou la basicité 
d'une eau naturelle, par la concentration en ions hydrogène libérés qu'elle contient. La détermination de l'équilibre acido-basique permet, grâce à sa grande sensibilité, de mettre en évidence et d'interpréter certaines des variations profondes qui se produisent. La mesure du $\mathrm{pH}$ est une opération indispensable. Nous constatons ici qu'il oscille entre 7,1. et 7,3 .

La résistivité électrique (RE), inverse de la conductibilité électrique (CE), rend possible l'appréciation de la minéralisation globale de l'eau; une eau offre d'autant plus de résistance au courant électrique qu'elle est moins chargée en sels minéraux dissous; certaines eaux des massifs cristallins des Vosges et de Bretagne ont une résistivité de l'ordre de 150000 à 160000 ohms $/ \mathrm{cm}$ à $20^{\circ} \mathrm{C}$. Au point de prélèvement, les valcurs citées sont comprises entre 20000 et $23000 \mathrm{ohms} / \mathrm{cm}$ à $20^{\circ} \mathrm{C}$, c'est-à-dire approximativement entre 21000 et $24000 \mathrm{ohms} / \mathrm{cm}$ à $18{ }^{\circ} \mathrm{C}$. A Sens, la RE est de l'ordre de 2500 à $3000 \mathrm{ohms} / \mathrm{cm}$ à $20^{\circ} \mathrm{C}$, c'est-à-dire de l'ordre de 2600 à $3150 \mathrm{ohms} / \mathrm{cm}$ à $18{ }^{\circ} \mathrm{C}$.

Le bas du tableau présente des courbes d'éléments chimiques: alcalinité, oxygène dissous, teneur en matières organiques (ou coefficient d'oxydabilité permanganique).

a) L'alcalinité traduit la teneur globale en carbonates et bicarbonates alcalins et alcalinoterreux. Elle est très faible ici, et, exprimée en $\mathrm{mg}$ de $\mathrm{CaO}$ (chaux anhydre) par litre, elle varie de 7 à $12 \mathrm{mg} / \mathrm{l}$; à Joigny, après que l'Yonne a reçu du Serein et de l'Armançon des eaux du sédimentaire, l'alcalinité oscille autour de 100 . Remarquons que les variations de la RE sont symétriquement opposées à celles de l'alcalinité.

b) Les quantités dosées d'oxygène dissous sont voisines de la valeur de saturation et peuvent dépasser la saturation; à cause du rôle que ce gaz joue dans le caractère agressif d'une eau chaude à l'égard des mẻtaux usuels, son dosage doit être pris en considération. Sa teneur diminue avec la pollution organique; mais il peut manquer dans les eaux souterraines profondes sans qu'il y ait pollution...

c) Enfin, l'évaluation de la teneur en matières organiques par le poids d'oxygène nécessaire à leur oxydation et emprunté au permanganate de potassium $\mathrm{MnO}_{4} \mathrm{~K}$ montre que la teneur est faible. Les variations sont intéressantes à observer: l'augmentation de substances organiques entraîne une diminution de l'oxygène dissous.

Au point de prélèvement considéré, les variations de débit ne sont pas normalement importantes; mais, dans un cours d'eau où les extremes sont marqués, la qualité de l'eau naturelle est très variable : à l'étiage, la charge en matières organiques est élevée; en période de hautes eaux, c'est la charge en argile colloildale qui est considérable. L'alcalinité, la teneur en O dissous, le développement des algues diverses subissent l'influence des conditions hydrologiques et saisonnières.

\section{CONCLUSION}

Ainsi, l'eau naturelle superficielle courante est une solution complexe et un milicu vivant en continuelle évolution. Cette inconstance des propriétés est un caractère fondamental de l'eau naturelle qui explique les observations scientifiques assidues auxquelles il nous faut nous livrer pour des raisons économiques : l'eau est une richesse minérale, une des plus grandes même, si ce n'est pas la plus grande. Aucune autre sans doute n'a autant d'utilisations pour ètre qualifiée d'essentielle.

Mais, en dehors de ce róle économique puissant, d'autres considérations, d'ordre historique et géographique, soulignent l'intérêt que nous lui portons. Bien que dépourvus de tout procédé d'analyse, les Anciens savaient empiriquement déceler la qualité des eaux pour leurs besoins. Si la véritable chimie des caux doil son essor, pour l'eau industrielle, a la production de la vapeur, el, pour l'cau potable, aux concentrations humaines qui sont nées des concentrations industrielles conséquentes, done si elle date du $\mathrm{x}^{\circ} \mathrm{x}^{\circ}$ siècle, nous présumons que l'intérêt des «chymistes» pour l'eau naturellc est antérieur : personnellement, nous considérons, en effet, comme les plus anciennes analyses de l'eau de Seine celles dont ont été chargés deux membres de l'Académie Royale des Sciences, en 1766; ils ont trouvé un résidu d'évaporation qui, après conversion des pintes en litres et des grains en milligrammes, nous donne des résultats voisins de ceux des mesures modernes [11].

De nos jours, "l'association des villes et des rivières » est, en France, un trait géographique qui subsiste [12] : l'attraction des eaux naturelles pour l'implantation des villes ne vous surprendra pas, quoique la juxtaposition villescours d'eau pérennes souffre bien des exceptions. Mais il est intéressant de noter que, le plus souvent, un site urbain lié à une riviere est un fait historique: site défensif pour une ville de méandre comme Besancon, pour une ville de fond de vallée comme Troyes; site de passage commercial comme Lutèce ou Angers: site de confluence comme Montereau ou Lyon; site de coude fluvial comme Orlíns ou Toulouse ou Bâle...

En m'appuyant sur les données récentes du recensement de 1962, j'ai calculé l'eftectif de la population urbaine de quatre départements, au nom fluvial, du bassin de la Seine: Seine-etMarne, Marne, Aube, Yonne. Puis, j'a determine l'effectif de cette population urbaine installée sur 


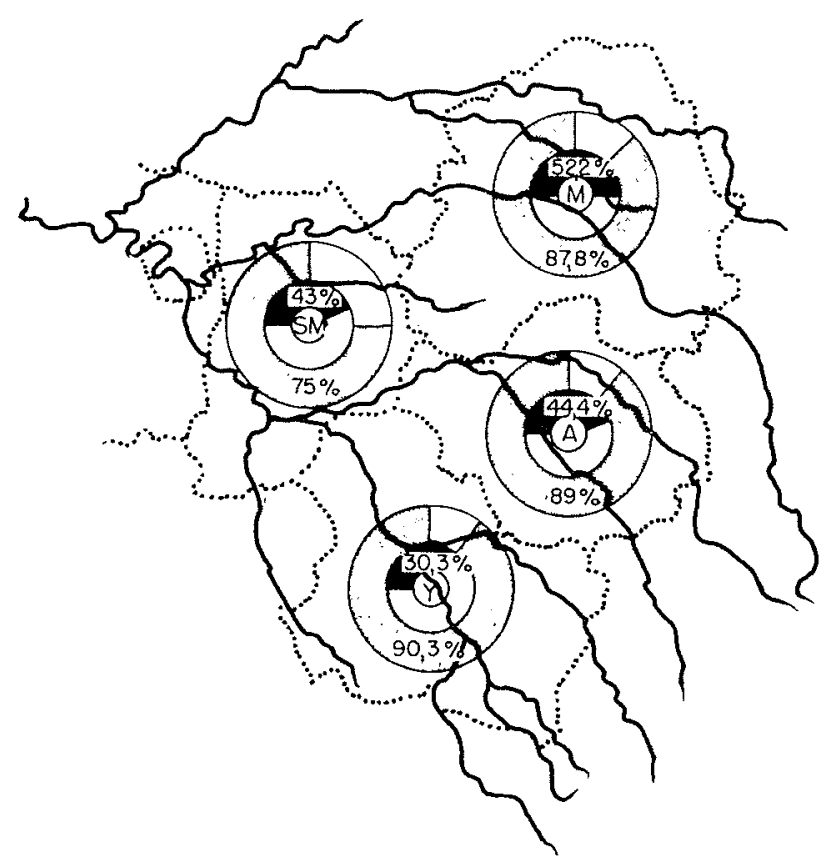

Por ropport à la population urbaine totale du département Par rapport à la population totale du département

FIG, 4

Attraction fluviale.

Pourcentages de population urbaine riveraine.

les hords d'une rivière (figurée sur la carte administrative de l'atlas Vidal de la Blache au $1 / 3800000^{\mathrm{e}}$ ) (fig. 4) :

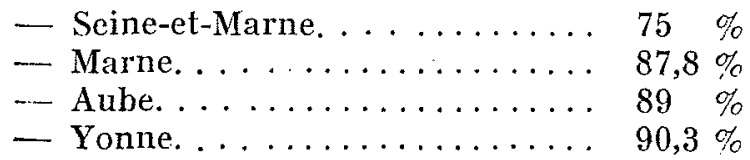

Enfin, j'ai calculé le \% de la population totale de chaque département que représente cette population urbaine près d'un cours d'eau.
D'après l'examen des résultats, il semble certain pour Seine-et-Marne, Marne et Aube, probable pour Yonne, que plus de la moitié de la population totale de chaque département est fixée non loin d'une eau naturelle courante.

Cette représentation cartographique exprime des caractères fondamentaux de l'eau naturelle superficielle courante : cette eau est intimement mêlée à l'existence comme à l'activité des hommes et elle acquiert une valeur sociale telle que se trouvent justifiées toutes les mesures prises pour assurer sa protection.

\section{RÉFÉRENCES BIBLIOGRAPHIQUES}

[1] M. Pardé. - Fleuves et rivières. Col. A. Colin, Paris, $1955,3^{\circ}$ edition.

[2] L. Gachon. - L'eau domestiquée, principe de la civilisation agronomique aussi bien qu'industrielle. Lib. Delaunay, Clermont-Ferrand, 1962.

[3] P. Geonge. - Précis de géographie économique. P.U.F., Paris, 1956.

[4] P. Koch. - L'alimentation en eau des agglomérations, Ed. Dunod, Paris, 1960.

[5] E. Lecherc et coll. - Dureté de l'eau. Belgique, Ed. Cebedoc, 1959, 2 vol.

[6] Standard Methods for the Examination of Water and Wastewater. New York, Amer. Public Health Assoc., 1960, $3^{\mathrm{e}}$ printing, January 1962.

[7] J. Bricard. - Physique des nuages. Paris, P.U.F., 1953.

[8] F. Borrel. - Sur les analyses de précipitations fractionnées et leur rapport avec la théorie de formation de la pluie. C.R. Acad. Sci., t. 244, 21 janvier 1957.

[9] Notes prises au cours du Professeur Plantefol. Voir aussi : R. Combes, La vie de la cellule végétale. Paris, Coll. A. Colin, 1946.

[10] P. Mangerel. - Le plancton dans les eaux de rivières destinées à l'alimentation. Cebedeau (trimestriel), $1957, \mathbf{n}^{\circ} 36$.

[11] M. M. Chartier. - Aspects de la connaissance qualitative, par le géographe, des eaux naturelles superficielles courantes. (A paraître).

[12] J. Blache. - Sites urbains et rivières françaises. Revue de Géographie de Lyon, 1959, $\mathrm{n}^{\circ} 1$.

\section{COMMENTAIRE}

Président : M. KocH

M. le Président remercie M. Chartier de son intéressante communication qui illustre l'insuffisance des points de vue habituellement admis et montre que toutes les caractéristiques de l'eau doivent être prises en considération dans les études sur l'état des rivières, notamment les données chimiques, biologiques, voire purement physiques comme la température.

M. le Président indique que, comme l'a suggéré M. Chartrer au cours de sa conférence, l'on peut s'associer au vœu que soit entreprise une recherche générale et une centralisation des relevés en ce qui concerne les eaux naturelles et cela pour deux raisons essentielles :

$1^{\circ}$ le relevé des analyses ne met en valeur que des points, et c'est le développement des programmes qui importe;

$2^{\circ}$ l'eau représente de plus en plus une matière première essentielle, du fait que les tonnages utilisés dépassent de loin ceux de toutes les autres matières premières quí ont la vedette et qu'elle ne comporte pas de succédané.

M. le Président propose en conséquence au Comité Technique de la S.H.F. d'appuyer le vou des géographes pour demander que des recherches systématiques soient poursuivies en France dans le domaine des eaux naturelles.

Le Comité Technique se range à cette proposition. 